\title{
Atypical case of lichen planus recognized by dermoscopy
}

\author{
Chrysoula Papageorgiou ${ }^{1}, Z$ oe Apalla ${ }^{1}$, Elizabeth Lazaridou ${ }^{1}$, Elena Sotiriou ${ }^{1}$, Efstratios Vakirlis ${ }^{1}$, \\ Demetrios Ioannides ${ }^{1}$, Aimilios Lallas ${ }^{1}$
}

1 First Department of Dermatology, Aristotle University, Thessaloniki, Greece

Key words: lichen planus; dermoscopy; psoriasis; Wickham striae; eczema

Citation: Papageorgiou C, Apalla Z, Lazaridou E, Sotiriou E, Vakirlis E, Ioannides D, Lallas A. Atypical case of lichen planus recognized by dermoscopy. Dermatol Pract Concept 2016;6(4):9. doi: 10.5826/dpc.0604a09

Received: August 8, 2016; Accepted: August 23, 2016; Published: October 31, 2016

Copyright: (92016 Papageorgiou et al. This is an open-access article distributed under the terms of the Creative Commons Attribution License, which permits unrestricted use, distribution, and reproduction in any medium, provided the original author and source are credited.

Funding: None.

Competing interests: The authors have no conflicts of interest to disclose.

All authors have contributed significantly to this publication.

Corresponding author: Chrysoula Papageorgiou, MD, First Department of Dermatology, Aristotle University, Thessaloniki, 124 Delfon Str, 54643, Thessaloniki, Greece. Tel.: 00302313308882; Fax: 00302310277979. Email: xrysapapageorgiou@gmail.com

ABSTRACT Lichen planus (LP) is an inflammatory disease that affects the skin-mainly the extremities and the trunk-the mucous membranes, the genitalia, the nails and the scalp. The diagnosis of LP is usually established clinically based on the typical morphology and distribution of the lesions in conjunction with the associated itch. We report a patient with LP manifesting highly psoriasiform lesions, that could only be correctly assessed after the application of dermoscopy, which revealed LP-specific findings.

\section{Introduction}

Lichen planus (LP) is a common inflammatory disease affecting the skin, the mucous membranes, the genitalia, the nails and the scalp [1]. Prevalence of lichen planus in the general population ranges from 0.1 to $4 \%$ and it is more common in females, especially in the perimenopausal period [1,2]. Pathophysiology of LP involves an immune-mediated reaction, in which an antigen is processed to T-lymphocytes and they, subsequently, attack basal keratinocytes, leading to apoptosis of the cells [3]. Several factors have been suggested as possible antigens, including viruses, bacterials and drugs [3,4].

The typical clinical manifestations of LP are purple to violaceous polygonal papules with sharp borders, usually pruritic, most commonly developing on the extremities and the trunk. Less frequently the disease affects the genital area, mucous membranes, palms and soles and nails [1,5]. Mucosal lesions are typified by the presence of reticular white lines, known as Wickham striae. The disorder has several clinical variations: annular, hypertrophic, atrophic, ulcerative, bullous, erythrodermic, inverse, linear, follicular, pemphigoides, pigmentosus, follicularis decalvans and actinic LP [3]. The diagnosis of LP is usually established clinically based on the characteristic morphology of the lesions and the coexisting intense pruritus. However, atypical presentations requiring histopathologic confirmation of the diagnosis do exist [1].

Dermoscopy allows the visualization of structures located in the epidermis, dermo-epidermal junction and papillary dermis that cannot be seen with the naked eye [6]. Initially, dermoscopy was almost exclusively used to evaluate skin 


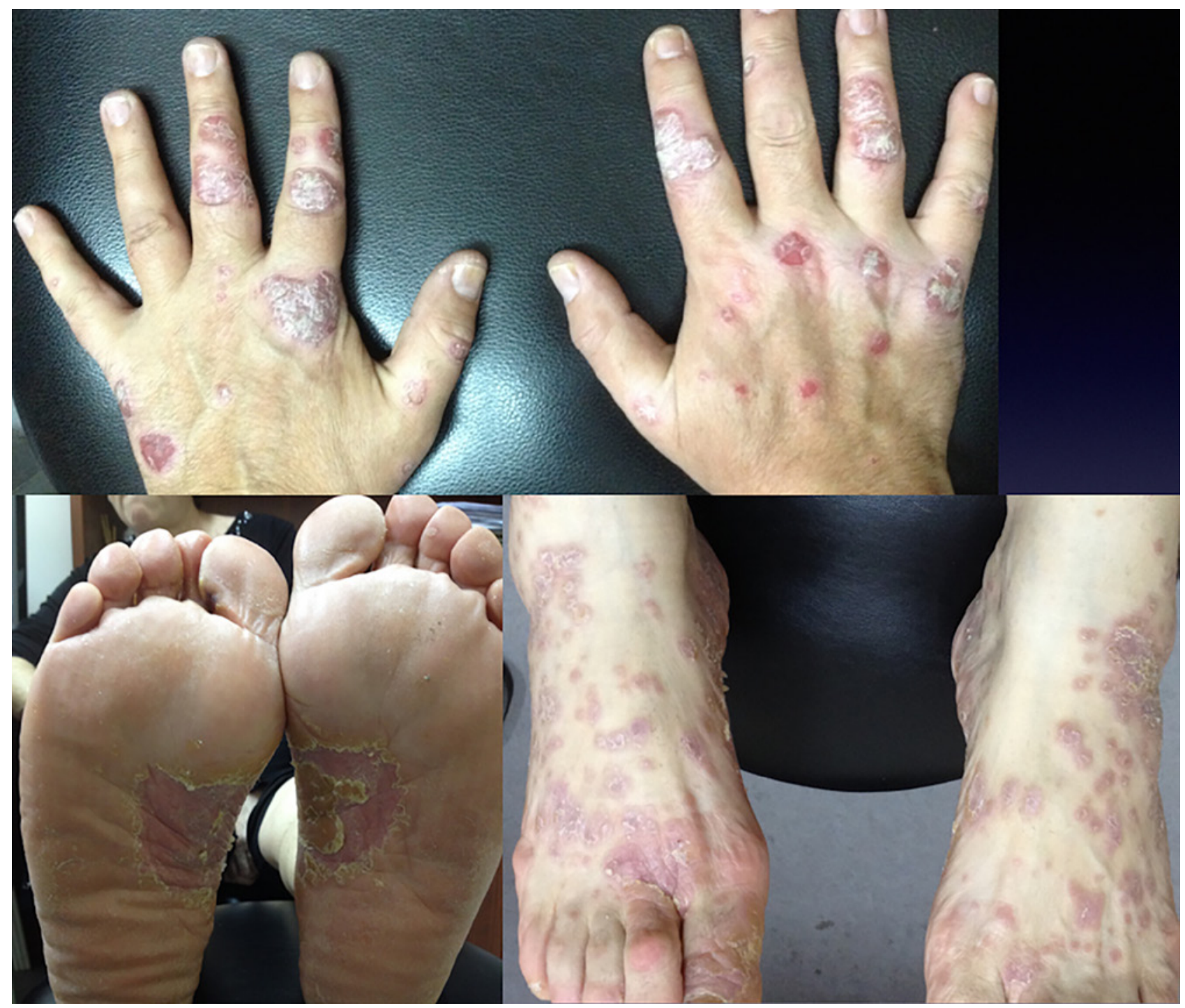

Figure 1. Lichen planus. Erythematous plaques covered by silvery-whitish scales. Both the clinical morphology and the distribution of the skin lesions are indicative of psoriasis. [Copyright: (2016 Papageorgiou et al.]

tumors $[7,8]$. However, cumulative evidence suggests that dermoscopy is also meaningful for the evaluation of inflammatory and infectious skin disorders [7,9]. In the field of papulosquamous dermatoses, dermoscopy has been shown to enhance the differential diagnosis among psoriasis, dermatitis, LP and pityriasis rosea [7,10]. Particularly for LP, dermoscopy brought to light that white crossing lines do not characterize only mucosal lesions, but cover virtually every cutaneous papule of active LP [7]. In this report we present a characteristic example of a patient with misleading clinical manifestations of LP resembling psoriasis. Application of dermoscopy was the key point guiding to the accurate diagnosis [11].

\section{Clinical presentation}

A 61-year-old woman visited our department for evaluation of a three-month, mildly pruritic eruption on the soles and the dorsal surfaces of the feet and hands. Clinical examination revealed hyperkeratotic plaques on the dorsal surface of the feet and hands and erythematous hyperkeratotic, partially erosive plaques on the soles. As shown in Figure 1, the overall clinical presentation was highly suggestive of psoriasis. Surprisingly, application of dermoscopy did not reveal the expected psoriatic pattern of regularly distributed dotted vessels and white scales (Figure 2). Instead, white crossing lines (the so-called Wickham striae) were dermoscopically evident, along with dotted and short linear vessels and yellow scales. Since the dermoscopic presence of Wickham striae is considered highly specific of LP, the dermoscopic findings prompted us to perform a biopsy for histopathologic assessment. Histopathology, as shown in Figure 3, revealed hyperkeratosis, dense hypergranulosis, vacuolar degeneration of basal cell keratinocytes, band-like lymphocytic infiltration in the upper dermis, as well as presence of colloid bodies, justifying the diagnosis of LP. 


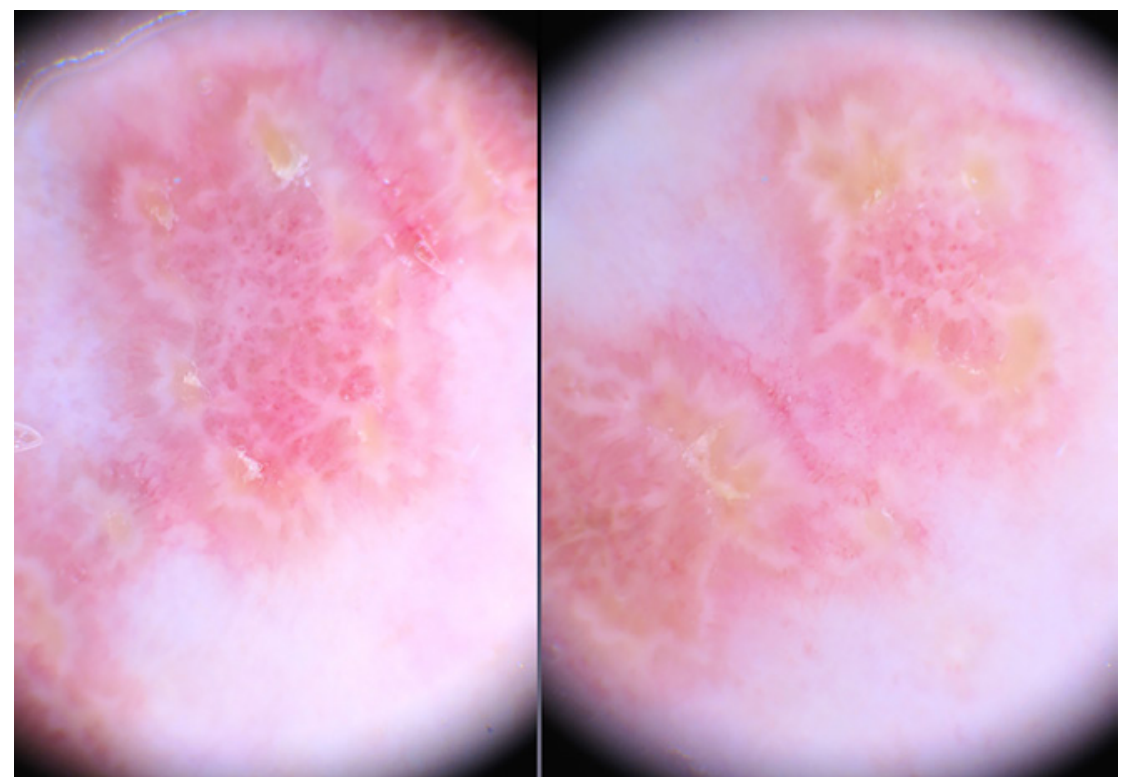

Figure 2. Lichen planus. Dermoscopy revealed dotted and short linear vessels and yellowish scales. However, the most prominent dermoscopic finding are the white crossing lines (Wickham striae). [Copyright: (92016 Papageorgiou et al.]

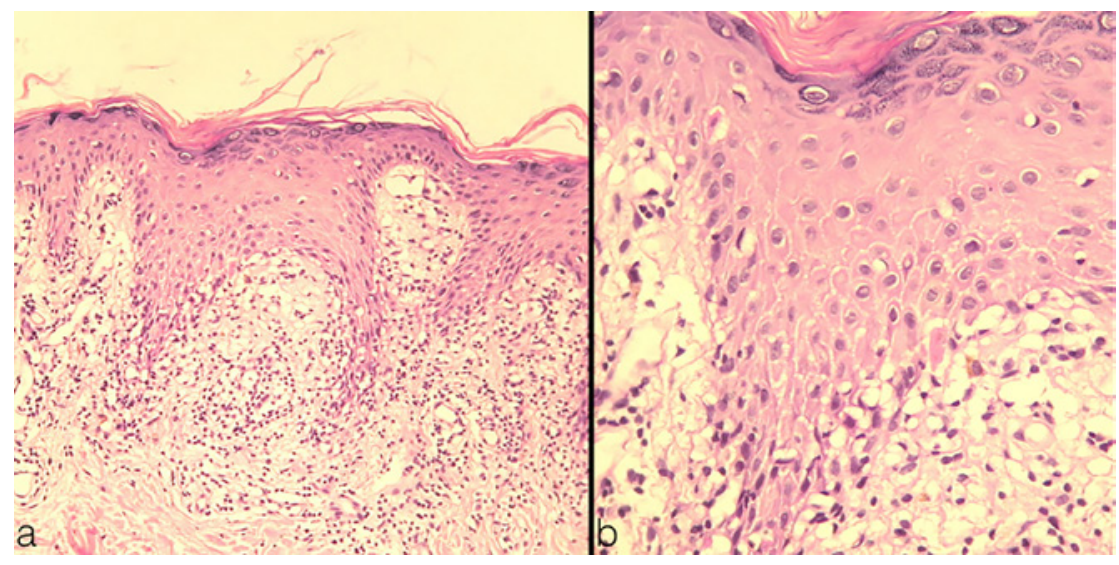

Figure 3. Lichen planus. Histopathology revealed hyperkeratosis, dense hypergranulosis, vacuolar degeneration of basal cell keratinocytes, band-like lymphocytic infiltration in the upper dermis, as well as presence of colloid bodies, justifying the diagnosis of lichen planus. [Copyright: @2016 Papageorgiou et al.]

\section{Discussion}

In the current case, clinical manifestations on the dorsal hands and plantar surfaces were highly suggestive of psoriasis, with eczema and LP included in the differential diagnosis. However, application of dermoscopy significantly influenced our diagnostic thoughts. This was because the lesion deviated from the standard dermoscopic pattern of psoriatic lesions, which are composed of regularly distributed dotted vessels and white-colored scales $[7,12,13]$. Although the presence of irregularly cal history, morphology and distribution often points towards a specific diagnosis [14]. LP and psoriasis are two characteristic examples, representing entities routinely diagnosed straightforward. However, equivocal clinical manifestations do exist in everyday practice, posing diagnostic doubts and often prompting clinicians to perform diagnostic biopsies [12]. It has been demonstrated that coupling clinical examination with dermoscopy significantly improves the diagnostic performance of clinicians [7].

However, in order to maximize the benefit from dermoscopy in differentiation of inflammatory dermatoses, clinicians have to virtually use their dermatoscope on every lesion. In daily routine, dermoscopy often confirms and strengthens our clinical suspicion. Furthermore, as shown in the current case, it may change our diagnostic thoughts, saving us from misdiagnosis and potential inappropriate management.

\section{References}

1. Usatine RP, Tinitigan M. Diagnosis and treatment of lichen planus. Am Fam Physician 2011;84(1):53-60. PMID: 21766756

2. Zakrzewska JM, Chan ES, Thornhill MH. A systematic review of placebocontrolled randomized clinical trials of treatments used in oral lichen planus. Br J Dermatol 2005;153(2):336-41. PMID: 16086745. DOI: 10.1111/j.13652133.2005.06493.x.

3. Wagner G, Rose C, Sachse MM. Clinical variants of lichen planus. J Dtsch Dermatol Ges 2013;11(4):309-19. PMID: 23320493. DOI: 10.1111/ddg.12031.

4. Lehman JS, Tollefson MM, Gibson LE. Lichen planus. Int J Dermatol 2009;48(7): 682-94. PMID: 19570072. DOI: $10.1111 /$ j.1365-4632.2009.04062.x.

5. Katta R. Lichen planus. Am Fam Physician 2000;61(11):3319-24. PMID: 10865927

6. Russo T, Piccolo V, Lallas A, Argenziano G. Recent advances in dermoscopy. F1000Res 2016;5. PMID: 26949523. DOI: 10.12688/f1000research.7597.1

7. Lallas A, Kyrgidis A, Tzellos TG, et al. Accuracy of dermoscopic criteria for the diagnosis of psoriasis, dermatitis, lichen 
planus and pityriasis rosea. Br J Dermatol 2012;166(6):1198-1205. PMID: 22296226. DOI: 10.1111/j.1365-2133.2012.10868.x.

8. Argenziano G, Soyer HP, Chimenti S, et al. Dermoscopy of pigmented skin lesions: results of a consensus meeting via the Internet. J Am Acad Dermatol 2003;48(5):679-93. PMID: 12734496. DOI: $10.1067 / \mathrm{mjd} .2003 .281$.

9. Zalaudek I, Argenziano G, Di Stefani A, et al. Dermoscopy in general dermatology. Dermatology 2006;212(1):7-18. PMID: 16319467. DOI: $10.1159 / 000089015$.

10. Vázquez-López F, Manjón-Haces JA, Maldonado-Seral C, Raya-Aguado C, Pérez-Oliva N, Marghoob AA. Dermoscopic features of plaque psoriasis and lichen planus: new observations. Dermatology 2003;207(2):151-6. PMID: 12920364. DOI: $10.1159 / 000071785$.

11. Friedman P, Sabban EC, Marcucci C, Peralta R, Cabo H. Dermoscopic findings in different clinical variants of lichen planus. Is dermoscopy useful? Dermatol Pract Concept 2015;5(4):51-5. PMID: 26693092. DOI: 10.5826/dpc.0504a13.
12. Lallas A, Apalla Z, Tzellos T, Lefaki I. Photoletter to the editor: Dermoscopy in clinically atypical psoriasis. J Dermatol Case Rep 2012;6(2):61-62. PMID: 22826724. DOI: 10.3315/ jdcr.2012.1102.

13. Lallas A, Apalla Z, Karteridou A, Lefaki I. Photoletter to the editor: Dermoscopy for discriminating between pityriasis rubra pilaris and psoriasis. J Dermatol Case Rep 2013;7(1):20-2. PMID: 23580911. DOI: $10.3315 /$ jdcr.2013.1131.

14. Lallas A, Argenziano G, Apalla Z, et al. Dermoscopic patterns of common facial inflammatory skin diseases. J Eur Acad Dermatol Venereol 2014;28(5):609-614. PMID: 23489377. DOI: 10.1111/ jdv.12146.

15. Lallas A, Apalla Z, Lefaki I, et al. Dermoscopy of early stage mycosis fungoides. J Eur Acad Dermatol Venereol 2013;27(5):61721. PMID: 22404051. DOI: 10.1111/j.1468-3083.2012.04499.x. 